\title{
An Evolutionary Intuitionistic Fuzzy K-means Clustering Approach based Cluster Head Selection in MANET
}

\author{
S. P. Swornambiga \\ Research Scholar, \\ Department of Computer Applications, \\ Manonmanium Sundaranar University, \\ Tirunelveli
}

\author{
Antony Selvadoss Thanamani, $\mathrm{PhD}$ \\ Associate Professor and Head \\ Research Department of Computer Science, \\ NGM College, Pollachi, India
}

\begin{abstract}
Recent technological advances in sensors, low-power microelectronics and miniaturization, and wireless networking enabled the design and proliferation of Mobile Adhoc Networks capable of autonomously monitoring and controlling environments. One of the most promising problems existing is efficient data transmission with less resource utilization between cluster head and base station. This paper handles the most influencing factor to obtain such efficiency is energy consumption, density and distance. This proposed work implements two stages in the Clustering phase the sensor nodes are clustered using the intuitionistic fuzzy Kmeans clustering using the membership and non-membership value of each factors taken into the account. Once clustering phase is over then the cluster head is selected based on the highest fitness function obtained using the genetic algorithm here the node with highest energy consumption and lower distance from base station and neighboring nodes are considered as optimal cluster head. The data packets are aggregated and transferred using the cluster head to the base station. During each round the cluster in reframed using intuitionistic fuzzy k-means. The result shows that the proposed method well performed in the case of uncertainty in cluster head selection
\end{abstract}

\section{Keywords}

MANET, cluster, Intuitionistic fuzzy K-means, uncertainty, energy consumption, distance

\section{INTRODUCTION}

The scalability of the MANET can be emphasized using the clustering of the node [1]. Electing a leader among the sensor node in manet for balanced load distribution is the process of cluster head selection and the elected node is called as Cluster Head. These head nodes preserve the information about the characteristics of its clusters which they belong to. The details of the nodes belong to the particular cluster and the path for traversing between these nodes is maintained as characteristics of the cluster information. The duty of the each $\mathrm{CH}$ node of various clusters it to be always communicative with their nodes belong to its own cluster. At the same time it must be reachable to other cluster nodes also using via the gateway or cluster head. Communication is done in three steps. First of all the cluster head receives the data sent by its members, secondly it compresses the data, and finally transmits the data to the base station or other $\mathrm{CH}$. Suitable cluster head can reduce energy utilization and enhances the network lifetime[2].

Electing a specific node as a cluster head is a very significant but complicated job. An assortment of factors can be considered for electing the best node as a cluster head [3]. Some of these factors consist of position of the node with respect to other nodes, mobility, energy, trust, and throughput of the node.

In general nodes of WSN and MANET have limited battery and resources. Process of election increases the overall processing overhead of the network. So the election process must also consider the processing and energy limitations of the nodes.

Only One cluster head per cluster must be chosen during an election process, because multiple cluster heads within a single cluster can give increase to cluster reformation, Quality of Service (QoS), and routing executive issues [4]. In the recent years, different surveys of $\mathrm{CH}$ election schemes were presented. Aspire of these surveys is to discuss their parameters, need of reclustering [5], and performance [6]. However to the best of knowledge, no overview of the $\mathrm{CH}$ election give emphasis to position of node in cluster, trust factor of nodes, and single cluster head selection per election process has been discussed so far.

In this paper, efforts have been made to develop a intuitionistic fuzzy based cluster structure formation with cluster head selection schemes are proposed in MANET. To have a better understanding, comparison of various $\mathrm{CH}$ selection techniques is made, in terms of parameters used and possibility of multiple $\mathrm{CHs}$ selection.

\subsection{Related Work}

There are several algorithms in the literature for cluster head election in mobile ad hoc networks some of them are discussed in this LEACH protocol is designed by [7] it is one of the mostly used hierarchical routing algorithms in the sensor networks. The main plan of LEACH protocol is to divide the total wireless sensor network into many clusters. The cluster head node is randomly selected; the chance of every node to be selected as cluster head is equal attributable to which energy consumption of whole network is averaged. Thus the LEACH will prolong the network life cycle. But due its random nature there is a high chance of choosing low energy node as cluster head and it also suffers from uneven distribution of nodes while forming clusters these factors will greatly affect the energy consumption.

In LEACH -C[8] Sensor nodes will communicate with the base station. Based on the information provided by the nodes the average node energy will be calculated. Sensor nodes which are having energy below this average value are not allowed to become cluster heads. To minimize the Energy required for normal nodes to the cluster head for data transmission BS will divide the network into required number of clusters.

In LEACH-F [9] clusters will be formed only once. rather the responsibility of cluster data gathering is rotated within the 
nodes. For Cluster formation LEAC - F depends on LEACH $\mathrm{C}$ algorithm.

Stable Election Protocol (SEP) is also a further modification to the LEACH protocol. In this protocol two types of nodes and two level hierarchies are considered. It is based on weighted electron probabilities of each node to become cluster head according to remaining energy in each node. SEP is a heterogeneous aware protocol and it prolongs the time interval before the death of first node which is referred as stability period [10]. In order to prolong stable region, SEP maintain a well balance of energy consumption.

The Lowest -ID [11, 12] algorithm also known as identifier based clustering, provide three different roles for the nodes: original, gateway and cluster head nodes. Periodically each node broadcast the list of $\mathrm{n}$ nodes with whom it can communicate including itself. Node with the minimum ID is selected as cluster head [13]. Thus the ids of the neighbors of the cluster head will be higher than that of the cluster head.

Highest - Degree [14, 13] also known as Connectivity based clustering, it was one of the first developed clustering algorithms used in ad hoc network. Similar to Lowest ID algorithm, a network consists of the two major components, Cluster Head and Member Node (Ordinary Node). Functionality of a cluster head node is to control the local traffic of the nodes in the cluster.

In this $\mathrm{K}$-hop connectivity ID clustering [15], it is the combination of LIC and HCC. HCC is take as first measure and LIC as the second measure. By flooding process, initially clustering request is sent to all nodes by a node. If only LIC is used, then it causes more number of cluster than needed, as a result the set of cluster head increases. If used only HCC, then it causes numerous ties between nodes. So when both cluster are used, it can limit on number of clusters by using HCC as first criterion that is the node having highest connectivity is selected as the cluster head, when number of hopes $\mathrm{k}=1$, connectivity it same as node degree.

In Mobility Based Metric for clustering algorithm (MOBIC) [16] it introduce a local mobility metric for the cluster formation process. Furthermore, cluster is formed in such away that mobile nodes with very low speed relative to their neighbors have the great chance to become cluster heads. The only difference between LIC and MOBIC is that in this cluster used mobility metric for cluster formation instead of ID information. In this algorithm, the received power levels of two successive Hello message transmissions are measured by each node from every neighbor. In Hello message every node broadcasts its own mobility metric to its 1-hop neighbors. And it is stored in the neighbor table of each neighbor with a break period

This paper aims to develop a dynamic energy efficient protocol architecture with Intuitionistic Fuzzy K-means based clustering on the basis of the uniform cluster location, the data transmission distances between the sensor nodes can be reduced by employing an evolutionary cluster head selection using genetic algorithm approach. The energy consumption is condensed, and the lifetime is extended for the sensor nodes by balancing the network load among the clusters.

\subsection{Materials and Methods}

\subsubsection{K-Means}

K-Means [10] is one of the classical methods for unsupervised evolutionary DM algorithm that solves the well-known clustering problem in a simple way and it's commonly used in medical imaging, biometrics and other emerging fields. It automatically adapts the best understood clustering methods for observing the grouping objects. The K-Means algorithm to form a group a collection of vectors into $\mathrm{K}$ groups and find a cluster center in each group. Finally got constant cluster center in each group in an iterative way that a cost function shown in (1) is minimized.

$$
J=\sum_{r=1}^{k} J_{r}=\sum_{r=1}^{k}\left(\sum_{x \in G_{r}} d\left(x, C_{r}\right)^{2}\right)
$$

Where, $\mathrm{k}$ is the number of clusters, $\mathrm{G}_{\mathrm{r}}$ and $\mathrm{C}_{\mathrm{r}}$ are the set of $\mathrm{rth}$ cluster and its associated center respectively. $X$ presents a data point and $d(a, b)$ is the distance functional (usually Euclidean distance) between $a$ and $b$.

In this method the value of $\mathrm{k}$ should be determined first. This value is fixed during the execution of the algorithm. The algorithm is summarized in the following steps:

Step1: Initializes the k cluster centers randomly

Step2: while the stop condition is not satisfied do the following:

a) Assign each vector to a cluster with nearest center.

b) Update cluster centers to the average of vectors belong to that cluster in order to decrease the cost function (2).

$$
\operatorname{Min}_{c_{k}} \sum_{i=1}^{N}\left\|x_{i}^{k}-C_{k}\right\|^{2}
$$

\subsubsection{Fuzzy K-Means}

Fuzzy K-Means (also called Fuzzy C-Means, Hard or Crisp Cluster) is a simplified extension of K-Mean Clustering algorithm [11, 12]. Identifying the pattern structure and attempt to capture \& quantifying the non-random imprecision are performed in this algorithm.

$$
J(K, m)=\sum_{k=1}^{K} \sum_{i=1}^{N}\left(U_{k_{i}}\right)^{m} d^{2}\left(x_{i}, c_{k}\right)
$$

Where $\mathrm{K}$ is the number of Cluster, $\mathrm{m}$ is the parameter, $\mathrm{U}_{\mathrm{k}_{\mathrm{i}}}$ Is the membership degree of $\mathrm{x}_{\mathrm{i}}$, in Cluster $\mathrm{K}, \mathrm{d}^{2}\left(\mathrm{x}_{\mathrm{i}}, \mathrm{C}_{\mathrm{k}}\right)$ is the distance from $\mathrm{x}_{\mathrm{i}}$ controls $\mathrm{c}_{\mathrm{k}}$. The parameter in this equation are the centroid vector $c_{k}$ and the components of the membership vector $U_{k_{i}}$. Evaluate $U_{k_{i}}$ shows the belonging ratio to a cluster $\mathrm{K}$ and centroid $\mathrm{c}_{\mathrm{k}}$ belonging expression cluster $\mathrm{k}$.

In this method a data point can belong to several groups with a membership degree between 0 and 1 which is shown by a matrix $U_{k \times m}$ where $k$ is number of clusters and $m$ is number of data points such that the condition in (4) is satisfied.

$$
\sum_{r=1}^{c} U_{r i}=1, \forall i=1, \ldots, n
$$

Where, $U_{r i}$ is the membership degree of ith pattern in rth cluster. The cost function used in this method is shown in the equation (5)

$$
J\left(U, c_{1}, \ldots, c_{k}\right)=\sum_{r=1}^{k} J_{r}=\sum_{r-1}^{k} \sum_{i}^{n} U_{r i}^{m} d\left(x_{i}, c_{r}\right)
$$


In order to minimize the cost function, membership degrees and cluster centers, in each iteration, are computed and the equations are (6) and (7)

$$
\begin{gathered}
U_{r i}=\frac{1}{\sum_{l-1}^{k}\left(\frac{d\left(x_{i}, c_{r}\right)}{d\left(x_{i}, c_{l}\right.}\right)^{2 /(m-1)}} \\
C_{r}=\frac{\sum_{i-1}^{k} u_{r i}^{m} x_{i}}{\sum_{j-1}^{k} u_{r j}^{m}}
\end{gathered}
$$

Where, $\mathrm{x}_{\mathrm{i}}$ represents the ith pattern in dataset. Like K-Means, Fuzzy K-Means [34] [39] works on those objects which can be represented in n-dimensional vector space and a distance measure is defined. This method performs the following steps.

Step1: Initializes the k fuzzy cluster centers randomly

Step2: while the stop condition is not satisfied do the following:

a) Compute the membership matrix $U$ according to the equation (6)

b) Update fuzzy cluster centers according to the equation (7)

\subsubsection{K-Modes}

To cluster features, the k-modes algorithm is utilized which is similar to the well known clustering method, k-means [22]. Mode of each cluster is defined as one of its features which have the largest multiple interdependence redundancy measure among other features in that cluster. The multiple inter-dependent redundancy measure is calculated for each feature by the following equation (8)

$$
\operatorname{MR}\left(A_{i}\right)=\sum_{A_{j} \in \text { Cluster }(i)} R\left(A_{i}: A_{j}\right)
$$

Where, Cluster (i) is the set of features which are in the same cluster with $\mathrm{Ai}$ and is the interdependence measure between the two features, $A_{i}$ and $A_{j}$, which is defined by the equation (9)

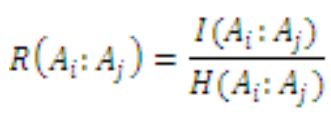

Where, $I\left(A_{i}: A_{j}\right)$ is the mutual information between $A_{i}$ and $A_{j}$ as computed in the equation (10)

$$
\begin{gathered}
I\left(A_{i}: A_{j}\right)=\sum_{k-1}^{m_{i}} \sum_{l-1}^{m_{j}} \operatorname{Pr}\left(A_{i}=v_{i k} \wedge A_{j}=v_{j l}\right) \\
\log \frac{\operatorname{Pr}\left(\mathrm{A}_{\mathrm{i}}=\mathrm{v}_{\mathrm{ik}} \wedge \mathrm{A}_{\mathrm{j}}=\mathrm{v}_{\mathrm{jl}}\right)}{\operatorname{Pr}\left(\mathrm{A}_{\mathrm{i}}=\mathrm{v}_{\mathrm{ik}}\right) \operatorname{Pr}\left(\mathrm{A}_{\mathrm{j}}=\mathrm{v}_{\mathrm{j} 1}\right)}
\end{gathered}
$$

$H\left(A_{i}: A_{j}\right)$ is joint entropy of $A_{i}$ and $A_{j}$ which is given by the equation (11)

$$
\begin{gathered}
H\left(A_{i}: A_{j}\right)=\sum_{k-1}^{m_{i}} \sum_{l-1}^{m_{j}} \operatorname{Pr}\left(A_{i}=v_{i k} \wedge A_{j}=v_{j l}\right) \\
\log \operatorname{Pr}\left(\mathrm{A}_{\mathrm{i}}=\mathrm{v}_{\mathrm{ik}} \wedge \mathrm{A}_{\mathrm{j}}=\mathrm{v}_{\mathrm{jl}}\right)
\end{gathered}
$$

$H\left(A_{i}: A_{j}\right)$ is used to normalize $I\left(A_{i}: A_{j}\right)$ in (10). The larger value for $I\left(A_{i}: A_{j}\right)$, the higher interdependency of two features, $A_{i}$ and $A_{j}$. Hence, there should be some pairs of values forthese features which are simultaneously visited with high frequency and other pairs are less probable. Therefore, having one of the values, other one may be approximated considering value pairs with high probability.

\subsubsection{Genetic Algorithm}

Genetic Algorithm (GA) is based on the random sampling techniques to find the sub-optimal solutions.GA have been widely used in high dimensionality problem such as micro array data.GA operators namely Selection, Mutation and Crossover based on new offering which is initiated by initial chromosomes as parents.GA generates the sample population with certain number of subset of features, is randomly selected from the data set. The 'goodness' of each subset of features is evaluated in terms of the fitness, which is estimated by a classification algorithm. The certain number of subset of features that meet the fitness criterion can be found using an iterative scheme. For each iteration, the fitness of each subset of feature is computed. The subset of feature is chosen for crossover and mutation based higher probability, to select the subset of features with better fitness, and versa. A new offspring (subset of features) is generated by making the crossover between two selected subset of features. After a crossover is performed, mutation is employed. Mutation changes randomly the new offspring in order to prevent falling all solutions in population into a local optimum. If the fitness of the subset of feature is satisfied, one near optimal subset of features is obtained, and a new population is generated. The computation is terminated until the predefined $\mathrm{K}$ near optimal subset of features is found.

\section{PROPOSED WORK}

This paper proposes an enhanced evolutionary model of energy-saving routing architecture was designed that clusters the sensor nodes based on the Intuitionistic Fuzzy C means based clustering algorithm. The main motive of this work is to reduce the energy consumption and to prolong the network lifetime in MANET in case of uncertainty in cluster head selection.

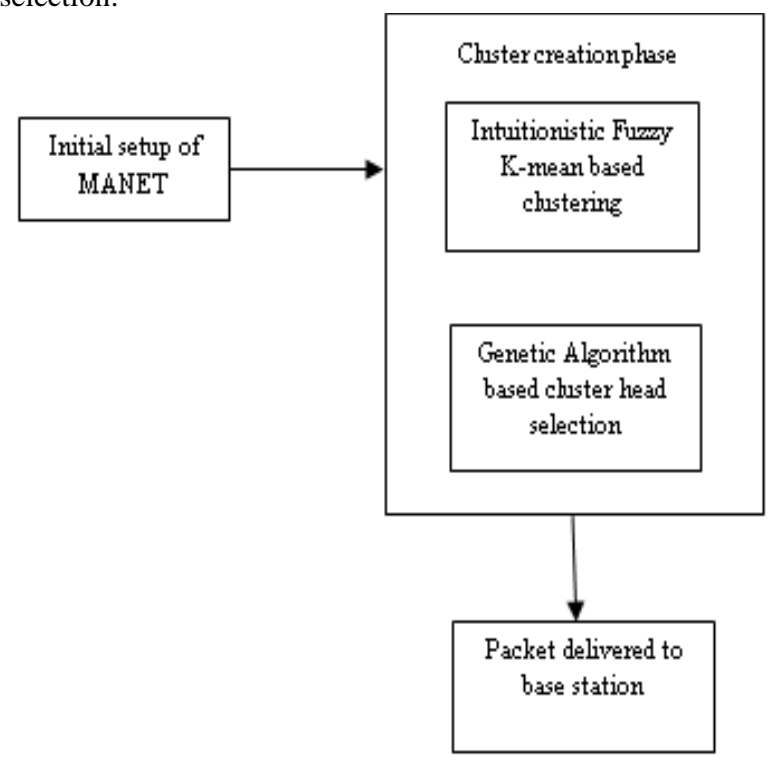

Fig. 1: Proposed Framework of An Evolutionary Intuitionistic Fuzzy K-Means based Cluster head Selection

\subsection{Intuitionistic Fuzzy K Means (IFKM)}

Intuitionistic fuzzy K-means clustering algorithm is based upon Intuitionistic fuzzy set theory. Fuzzy set generates only membership function $\mu(\mathrm{x}), \mathrm{x} \in \mathrm{X}$, whereas Intuitionistic fuzzy set (IFS) given by Atanassov [17] considers both membership $\mu(\mathrm{x})$ and non-membership $v(\mathrm{x})$. An Intuitionistic fuzzy set $\mathrm{A}$ in $\mathrm{X}$, is written as the equation (12)

$$
A=\left\{x, \mu_{A}(x), v_{A}(x) \mid x \in X\right\}
$$


Where $\mu_{\mathrm{A}}(\mathrm{x}) \rightarrow\lfloor 0,1\rfloor, \mathrm{v}_{\mathrm{A}}(\mathrm{x}) \rightarrow\lfloor 0,1\rfloor$ are the membership and non-membership degrees of an element in the set A with the condition as mentioned in the equation (13)

$$
0 \leq \mu(x)+v_{A}(x) \leq 1
$$

When $\mu \mu_{A}(x) \rightarrow[0.1], v_{A}(x) \rightarrow[0.1]$ for every $x$ in the set $A$, then the set A becomes a fuzzy set. For all Intuitionistic fuzzy sets, Atanassov also indicated a hesitation degree, $\pi_{\mathrm{A}}(\mathrm{x})$ which arises due to lack of knowledge in defining the membership degree of each element, $\mathrm{x}$ in the set $\mathrm{A}$ and is given by the equation (14)

$$
\begin{gathered}
\pi_{\mathrm{A}}(\mathrm{x})=1-\mu_{\mathrm{A}}(\mathrm{x})-\mathrm{v}_{\mathrm{A}}(\mathrm{x}) ; 0 \leq \pi_{\mathrm{A}}(\mathrm{x}) \\
\leq 1
\end{gathered}
$$

Due to hesitation degree, the membership values lie in the interval as mentioned in the equation (15)

$$
\left[\mu_{A}(x), \mu_{A}(x)+\pi_{A}(x)\right]
$$

IFKM minimizes the objective function as in equation (16)

$$
\begin{gathered}
J_{I F K M}=\sum_{r=1}^{k} J_{r} \\
\sum_{r=1}^{k} \sum_{i=1}^{n} \mu_{r i}^{* m} d\left(x_{i}, c_{r}\right)^{2} \\
+\sum_{r=1}^{k} \pi_{r}^{*} e^{1-\pi_{r}^{*}}
\end{gathered}
$$

Where,

$$
\mu_{r i}^{*}=\mu_{r i}+\pi_{r i}
$$

where $\mu_{\mathrm{ri}}^{*}$ denotes the Intuitionistic fuzzy membership and $\mu_{\mathrm{ri}}$ denotes the conventional fuzzy membership of the ith feature in rth cluster defined as follows in the equation (17)

$$
U_{r i}=\frac{1}{\sum_{l-1}^{k}\left(\frac{d\left(x_{i}, c_{r}\right)}{d\left(x_{i}, c_{l}\right.}\right)^{2 /(m-1)}}
$$

This paper adopted the distance measure proposed by D. M. Tsai and C. C. Lin. they incorporated a new distance measure into the conventional FCM as equation (19)

$$
d\left(x_{i}, c_{r}\right)=\frac{\left\|x_{i}-c_{r}\right\|^{2}}{\sigma_{r}}
$$

and $\sigma$ is the weighted mean distance of cluster $r$ and is given by equation (20)

$$
\sigma_{r}=\left\{\frac{\sum_{i=1}^{n} \mu_{r i}^{* m} \cdot\left\|x_{i}-c_{r}\right\|^{2}}{\sum_{i=1}^{n} \mu_{r i}^{* m}}\right\}
$$

$\pi_{\mathrm{ri}}$ is hesitation degree, which is defined as follows in the equation (21)

$$
\pi_{r i}=1-\mu_{r i}-\left(1-\mu_{r i}^{\alpha}\right)^{1 / \alpha}, \quad \alpha>0
$$

and is calculated from Yager's Intuitionistic fuzzy complement, the Intuitionistic fuzzy set becomes(22)

$$
\begin{gathered}
A^{I F S}=\left\{x, \mu_{A}(x),\left(1-\mu_{A}(x)^{\alpha}\right)^{1 / \alpha} \mid x \in X\right\} \\
\pi_{r}^{*}=\frac{1}{N} \sum_{i=1}^{n} \pi_{r i}
\end{gathered}
$$

Step1: Initializes the $\mathrm{k}$ Intuitionistic fuzzy cluster centers randomly

Step2: while the stop condition is not satisfied do the following:

a) Compute the Intuitionistic Fuzzy membership and indeterministic value of each data point according to the equations (17) and (23)

b) Update Intuitionistic fuzzy cluster centers according to equation (20)

For each node features with different membership and indeterministic values assigned to $\mathrm{k}$ different clusters. Because in Intuitionistic fuzzy each feature may not belong to just one cluster and it is much better to, consider the correlation of each feature to features in entire clusters.

Hence, during the selection of the best $\mathrm{CH}$ node depends on its features namely energy, density, distance of neighbor nodes, more accurate relations between genes are available. The main point here is that in selecting each feature, it is considered among the entire clusters not just one. Hence, in this case a feature, which is not, correlated enough with members of one cluster but its correlation among entire clusters is high, gains more chance to be selected in comparison with crisp attribute clustering techniques.

This method uses the interdependence redundancy measure as the similarity measure. To cluster features, used the Intuitionistic k-modes algorithm in which mode of each cluster is defined as one of its features which has the largest multiple interdependence redundancy measure among other features in that cluster.

A modified version of (6), where distance functional is defined as the inverse of interdependency measure

$$
\begin{aligned}
& \mu_{r i}^{*} \\
& =\frac{1}{\sum_{i=1}^{k}\left[\frac{R\left(A_{i}, n_{l}\right)}{R\left(A_{i}, n_{r}\right)}\right]^{2 / m-1}} \\
& +\left[1-\frac{1}{\sum_{i=1}^{k}\left[\frac{R\left(A_{i}, n_{l}\right)}{R\left(A_{i}, n_{r}\right)}\right]^{2 / m-1}}\right. \\
& \left.-\left[1-\left[\frac{1}{\sum_{i=1}^{k}\left[\frac{R\left(A_{i}, n_{l}\right)}{R\left(A_{i}, n_{r}\right)}\right]^{2 / m-1}}\right]^{\alpha}\right]^{1 / \alpha}\right]
\end{aligned}
$$

Where, li s number of clusters. $\mu_{\mathrm{ri}}^{*}$ is Intuitionistic fuzzy membership degree of ith feature in rth cluster and $\mathrm{m}$ is a weighting expoent. Afterwards, to satisfy the condition (4) $\mu_{\mathrm{ri}}^{*}$ is normalized by equation (25)

$$
\mu_{r i}^{*}=\frac{\mu_{r i}^{* \text { new }}}{\sum_{l=1}^{k} \mu_{l i}^{* \text { old }}}
$$

In this approach mode of a cluster is updated to the feature with the highest fuzzy multiple interdependence redundancy in that cluster. Fuzzy multiple interdependence redundancy of each feature should be calculated regardless of its own membership degree in associated cluster. Considering this Intuitionistic fuzzy membership degree, mode of each cluster will never change, since it has high membership degree in that cluster. 


\subsection{Novel Genetic algorithm based Intuitionistic fuzzification techniques in selection of cluster heads from clusters}

After Clustering of nodes, the best node is selected based on the optimal value of energy, density, centrality and the distance between nodes one should be selected from each cluster. In this paper genetic algorithm technique is proposed in order to consider Intuitionistic fuzzy memberships of attributes of nodes in clusters during selection. At first each feature is consider to belong to a cluster which has highest membership degree. Members of rth cluster are represented by member r. Rank of each feature in its cluster is calculated and the one with the highest ranks are selected as the candidate. For calculating the rank of each feature in /Intuitionistic fuzzy approach technique assigns a positive weight to the inter-dependency of features which are belonged to membership to cluster $r$. It also assigns a negative weight as a penalty to the interdependency of features as non membership to cluster $r$. The assigned weight is equal to the membership degree of the feature in its cluster.

\subsection{Set-up Phase:}

First phase is the set-up phase and it is performed only one time.

- In the set-up phase, pre-defined numbers of sensor nodes are chosen as cluster heads.

- The number of $\mathrm{CHs}$ also indicates the number of clusters in the network.

- Intuitionistic fuzzy K-means based Cluster formation

- Once the Initial Clusters heads are selected and framed clusters then each Non-CH nodes are assigned to the clusters based on Intuitionistic fuzzy K-Means clustering

- The non-CH nodes with similar range of membership and non membership values are assigned to different clusters based on the features of residual energy, density, centricity and distance.

- Once Cluster formation is completed then the optimal cluster head is selected

- Steady State Phase based on Genetic Algorithm based Cluster head Selection

- In this phase the potential cluster head is selected using genetic algorithm.

- In the method, GA is used to maximize the lifetime of the network by means of rounds. Binary representation of the network is used and each sensor node corresponds a bit. $\mathrm{CHs}$ are represented as " 1 " and non- $\mathrm{CH}$ nodes are represented as " 0 ".

- The representation of a network is called a Chromosome or Genome, a collection of bits. Initially the GA starts with a population, a predefined number of chromosomes, consists of randomly generated individuals.

- Then GA evaluates each chromosome by calculating its fitness. Fitness of a chromosome depends on some fitness parameters described as follows

- After evaluating the fitness of each chromosome in the population, GA selects the best fit chromosomes by using a specific selection method based on their fitness values and then applies two operators, Crossover and Mutation, respectively. These operations are carried out to produce a new population better than the previous one for the next generation.

- The Cluster Head for each round is selected the node with highest fitness value ie the residual energy and distance from it neighbor nodes are considered.

- The elected $\mathrm{CH}$ node once it receives messages from all member nodes, it fuses the data packets into one packet and sends it to the base station (BS).

- When all CHs send their data to BS, a round is completed.

- At the end of each round the BS checks the fitness function (energies) of $\mathrm{CHs}$ and the member nodes. If the energy of a $\mathrm{CH}$ is under the average energy of the member nodes of its cluster then the reclustering is performed using intuitionistic fuzzy $\mathrm{K}$ means

- This round is completed, and the next round begins with a set-up phase and a steady-state phase; this process is repeated.

\section{EXPERIMENTAL RESULT}

This section describes the proposed work simulation environment developed using matlab and illustrates the simulation results, and the proposed scheme is compared with LEACH and SEP. To prove that the proposed scheme is promising, this work designed a visual interface simulator and implements several existing schemes for fair comparison. The assumptions for our simulation environment are as follows:

- A BS is fixed and located far from the sensor nodes.

- The initial location of each user is randomly distributed in the sensing area.

- The speed of each user is uniformly distributed between 0 and $5 \mathrm{~km} / \mathrm{h}$.

- All of the sensor nodes can send data to the BS.

- All of the sensor nodes in the network are homogeneous and energy constrained.

- The non-CH nodes can monitor the environment and directly send data to the $\mathrm{CH}$ node, or through the non$\mathrm{CH}$ hop nodes.

- The CH node can gather data, compress it, and forward it to the BS.

- The initial energy is the same for each sensor node.

- The energy of the sensor node cannot be recharged. The sensor node continues to sense and transfer data until its energy is depleted.

All of the parameters used in this simulation are listed below:

* 100 nodes are randomly distributed in 50x50 square meters area

* BS is located 100 meters away from the network.

* Initial Energy is .5 Joules

* The radio dissipates Eelec $=50 \mathrm{~nJ} / \mathrm{bit}$ to run the transceiver or receiver and $\in \mathrm{amp}=100 \mathrm{pJ} / \mathrm{bit} / \mathrm{m} 2$ for transmit amplifier

* No. of Round is 9000

The performance of using a Evolving Intuitionistic Fuzzy K means based cluster head selection approach can be clearly seen in Figure 2, 3 and 4. The proposed method outperforms LEACH and SEP Schemes

The figure 2 shows the performance of LEACH, SEP and EIFKMACH schemes based on number of rounds vs dead nodes. The number of rounds varies from 1000 to 9000 . Each rounds the dead nodes are determined for each scheme and the result shows the lower number of cluster head nodes found 
dead in proposed Scheme comparing to other existing scheme used in this work. This due to the selection of cluster head with highest residual energy with the help of intuitionistic fuzzy K-means based clustering and selection of highest fitness value based nodes as cluster heads in MANET

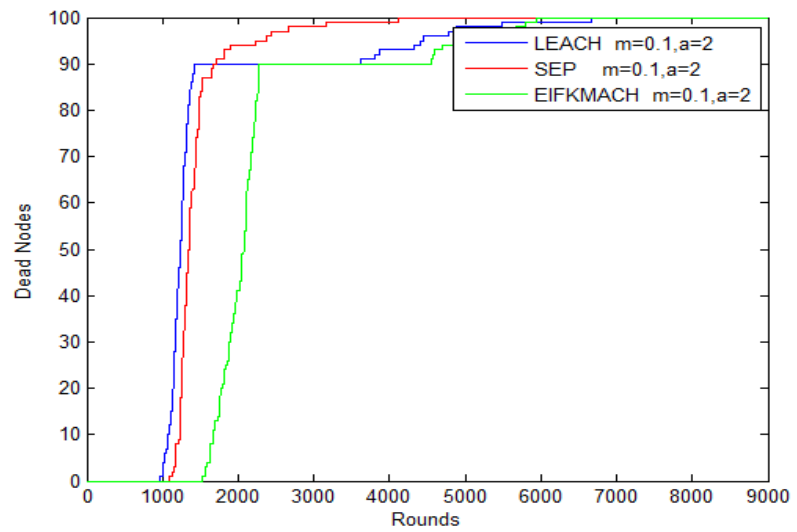

Fig 2

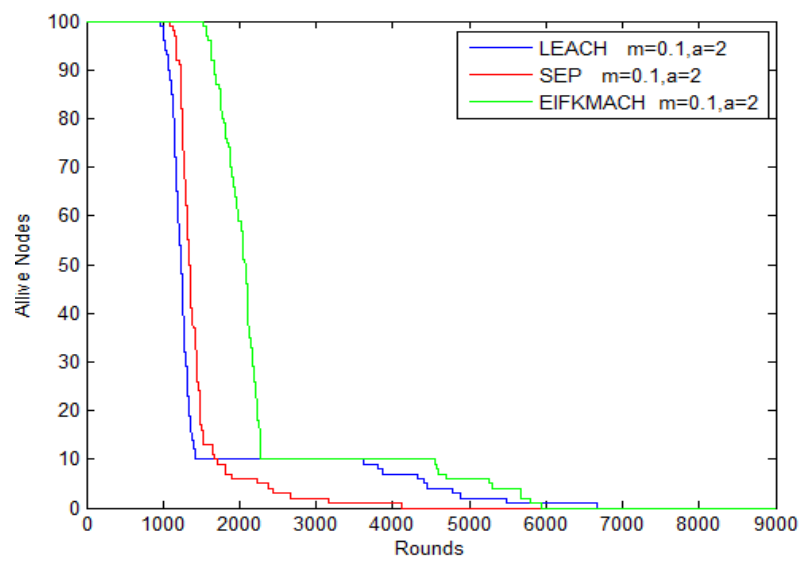

Fig. 3

The figure 3 shows the performance of LEACH, SEP and EIFKMACH schemes based on no of rounds versus number of alive nodes. The number of rounds varies from 1000 to 9000. In each round alive nodes are determined for each scheme and the result shows the increase number of cluster head nodes prolonged life time in proposed Scheme comparing to other existing scheme used in this work. This due to the selection of cluster head with lowest member ship value of distance from the base station to cluster head and the non cluster head density with the help of intuitionistic fuzzy $\mathrm{K}$-means based clustering and selection of highest fitness value based nodes as cluster heads in MANET.

The figure 4 shows the performance of LEACH, SEP and EIFKMACH schemes based on number of packet delivered to base station from the cluster head. The number of rounds varies from 1000 to 9000 . The packet delivery ration of the each scheme is determined and the result shows that the proposed work deliveries more number of packets to the based station due to the optimal selection of cluster head. This due to the selection of cluster head with lowest member ship value of distance from the base station to cluster head and the non cluster head density with the help of intuitionistic fuzzy $\mathrm{K}$-means based clustering and generation of promising nodes as population in each round, election of cluster head with highest fitness value with the distance as the factor.

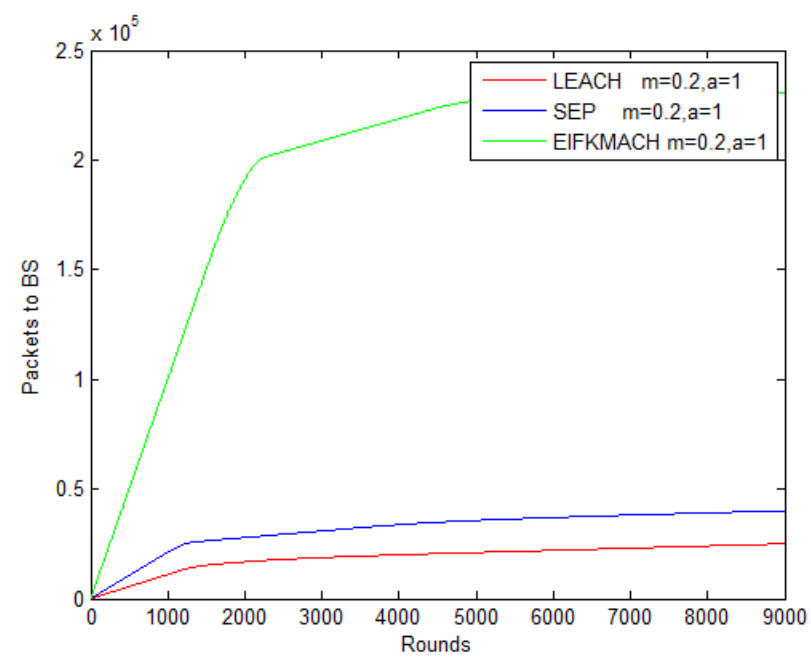

Fig. 4

\section{CONCLUSION}

Energy consumption in a mobile Adhoc networks can be due to either useful or wasteful work. Useful energy consumption results from transmitting and receiving data packets, querying requests, and forwarding data. Wasteful energy consumption is due to collisions and resulting retransmissions, idle listening to the channel, and overhead of each packet header. Simulation results indicate that the proposed algorithm EIFKMACH achieves lower energy consumption and prolonged network lifetime in MANET. In the proposed scheme, an intuitionistic fuzzy $\mathrm{k}$-means based clustering architecture for sensor nodes is created. The election of cluster head is done through the genetic algorithm with the factors of residual energy, distance and density of each node. The node with highest factor is selected based on the membership and non-membership value of the factors. The data passed by the nodes in the cluster doesn't communicate with the base station they pass it to the cluster head where the data are collected, compressed and passed to the base station via gateway or the other cluster heads. Thus the proposed work best suits in adversial environment.

This work can be extended in future by enhancing the selection process of cluster head with additional parameters, choosing different types of clustering formats in dynamic and adversial environment, determining misbehaving nodes which leads to node failure. For optimal selection process to elect the cluster head the other optimal algorithms like bat, swarm optimization, ant colony can be used.

\section{REFERENCES}

[1] C. R. Lin and M. Gerla, "Adaptive Clustering for Mobile Wireless Networks," IEEE JSAC, vol. 15, Sept. 1997, pp. 1265-75.

[2] R. Agarwal and D. Motwani, "Survey of clustering algorithms for MANET," http://arxiv.org/abs/0912.2303.

[3] M. Chatterjee, S. Sas, and D. Turgut, "An on-demand weighted clustering algorithm (WCA) for ad hoc networks," in Proceedingsf the IEEE Global Telecommunications Conference(GLOBECOM '00), 2000.

[4] P. Chatterjee, "Trust based clustering and secure routing scheme for mobile ad hoc networks," International Journal of Computer Networks and Communication, vol. 1, no. 2, pp. 84-97, 2009. 
[5] S. Chinara and S. K. Rath, "A survey on one-hop clustering algorithms in mobile ad hoc networks," Journal of Network andSystems Management, vol. 17, no. 1-2, pp. 183-207, 2009.

[6] C.-L. Fok, G.-C. Roman, and C. Lu, "Rapid development and flexible deployment of adaptive wireless sensor network applications," in Proceedings of the 25th IEEE International Conference on Distributed Computing Systems (ICDCS '05), pp. 653-662, June 2005.

[7] C. Liu, C. Lee, L.ChunWang, "Distributed clustering algorithms for data gathering in wireless mobile sensor networks", Elsevier Sci. J. Parallel DistribComput., Vol.67, 2007, pp.1187 -1200

[8] W. Heinzelman, A. Chandrakasan and H. Balakrishnan., "An Application -Specific Protocol Architecture for Wireless Microsensor Networks", IEEE Trans. Wireless Communications, Vol. 1, No.4, October 2002, pp.660670.

[9] W. Heinzelman, Application - Specific Protocol Architectures for Wireless Networks, Ph.D Thesis, Massachusetts Institute of Technology, June 2000.

[10] G. Smaragdakis, I. Matta, A. Bestavros, SEP: A Stable Election Protocol for clustered heterogeneous wireless sensor networks, In Second International Workshop on Sensor and Actor Network Protocols and Applications (SANPA), 2004

[11] D. J. Baker and A. Ephremides. A Distributed Algorithm for Organizing Mobile Radio Telecommunication Network. In proceedings of the $2^{\text {nd }}$ International Conference on Distributed Computer Systems, page 476 - 483, April 1981.

[12] D. J. Baker and A. Ephremides. The Architectural Organization of a Mobile Radio Network via a Distributed Algorithm. IEEE Transactions on Communication, COM - 29 11:1694, 1701. November 1981
[13] Ratish Agrawal, Dr. Mahesh Motwani, "Survey of Clustering Algorithm for Mobile Ad hoc Network", IJCSE, Vol. 1 Issue 2, pp. 98-104, 2009

[14] M. Gerla and J. T. Tsai, "Multicluster, Mobile, Multimedia Radio Network. Wireless Network," 1995

[15] G. Chen, F. Nocetti, J. Gonzalez, and I. Stojmenovic, "Connectivity based $\mathrm{k}$ - hop clustering in wireless network," In proceeding of International Conference on System Science, Vol. 7, pp. 188.3, 2002

[16] P. Basu, N. Khan, and T.D.C. Little, "A Mobility Based Metric for Clustering in Mobile Ad Hoc Networks", In proceeding of IEEE ICDCSW, pp. 413 - 18, Apr. 2001

[17] Ching-Wen Huang, Kuo-Ping Lin, Ming-Chang Wu, Kuo-Chen Hung, Gia-Shie Liu, Chih-Hung Jen, Intuitionistic fuzzy c-means clustering algorithm with neighborhood attraction in segmenting medical image, Soft Computing. February 2015, Volume 19, Issue 2, pp $459-470$

[18] Krassimir T. Atanassov, 1986,"'ntuitionistic Fuzzy Sets", Fuzzy Sets and Systems, Vol.20, pp.87-96.

[19] Chinatsu Arima, Taizo Hanai and Masahiro Okamoto, 2003," Gene Expression Analysis Using Fuzzy K-Means Clustering”, Genome Informatics, Vol.14(1), pp. 334335 .

[20] E.W. Forgy (1965). "Cluster analysis of multivariate data: efficiency versus interpretability of classifications". Biometrics. 21: 768-769. JSTOR 2528559.

[21] J. C. Dunn (1973): "A Fuzzy Relative of the ISODATA Process and Its Use in Detecting Compact WellSeparated Clusters", Journal of Cybernetics 3: 32-57

[22] J. C. Bezdek (1981): "Pattern Recognition with Fuzzy Objective Function Algorithms", Plenum Press, New York $\square$ Tariq Rashid: "Clustering" 